\title{
Effects of the brief viewing of emotional stimuli on understanding of insight solutions
}

\author{
Michiko Sakaki • Kazuhisa Niki
}

Published online: 9 August 2011

(C) Psychonomic Society, Inc. 2011

\begin{abstract}
In the present study, we examined whether and how brief viewing of positive and negative images influences subsequent understanding of solutions to insight problems. For each trial, participants were first presented with an insight problem and then briefly viewed a taskirrelevant positive, negative, or neutral image $(660 \mathrm{~ms})$, which was followed by the solution to the problem. In our behavioral study (Study 1), participants were faster to report that they understood the solutions following positive images, and were slower to report it following negative images. A subsequent fMRI study (Study 2) revealed enhanced activity in the angular gyrus and medial prefrontal cortex (MPFC) while viewing solutions following positive, as compared with negative, images. In addition, greater activation of the angular gyrus was associated with more rapid understanding of the solutions. These results suggest that brief viewing of positive images enhances activity in the angular gyrus and MPFC, which results in facilitation of understanding solutions to insight problems.
\end{abstract}

Keywords Positive emotion · Insight - Valence · Mood and cognition · fMRI

\footnotetext{
M. Sakaki

University of Southern California,

3715 McClintock Ave.,

Los Angeles, CA 90089, USA

e-mail: msakaki@usc.edu

K. Niki $(\bowtie)$

National Institute of Advanced Industrial Science and Technology,

1-1 Umezono,

Tsukuba, Ibaraki 305-8568, Japan

e-mail:k2ki22@gmail.com
}

The ability to think in a flexible manner is a critical component of creativity. Past research has revealed that positive and negative mood states have opposing influences on cognitive flexibility. For example, people show enhanced cognitive flexibility in categorization (e.g., Isen \& Daubman, 1984) and improved performance in creative problem solving (e.g., Isen, Daubman, \& Nowicki, 1987; Rowe, Hirsh, \& Anderson, 2007) after positive mood induction (e.g., generating positive thoughts for $10 \mathrm{~min}$ or viewing comedy films). In contrast, cognitive flexibility is impaired by situational stressors (e.g., giving a public speech or taking a mathematical test; Alexander, Hillier, Smith, Tivarus, \& Beversdorf, 2007; Beversdorf, Hughes, Steinberg, Lewis, \& Heilman, 1999) and sustained negative mood states (Subramaniam, Kounios, Parrish, \& Jung-Beeman, 2009; Vosburg, 1998).

Although most previous studies have targeted sustained mood states lasting several minutes, recent research has revealed that subjective feelings of emotion can occur very quickly after encountering emotional stimuli (e.g., within $500 \mathrm{~ms}$; Rudrauf et al., 2009). These findings suggest the possibility that cognitive flexibility is affected not only by sustained mood states, but also by transient short-term emotional states induced by brief presentation of emotional stimuli. To address this possibility, in the present study, we examined whether and how the brief viewing of positive and negative stimuli influences understanding of solutions to insight problems.

\section{Mood and insight problem task}

One of the tasks frequently used to examine the effects of mood on cognitive flexibility has been the insight problem 
task (for reviews, see Baas, De Dreu, \& Nijstad, 2008; Davis, 2009). Insight occurs when people reconstruct problems and form novel, task-related representations (Bowden, Jung-Beeman, Fleck, \& Kounios, 2005; JungBeeman et al., 2004; Luo \& Knoblich, 2007; Weisberg, 1996). Consider the following problem (the Marriage problem): "A man in a small town married 20 different women from the same town. All of them are still living and he never divorced. Polygamy is unlawful, but he has broken no law. How can this be?" First, people tend to interpret "to marry" as meaning that a person takes someone in marriage. However, to solve this problem, they need to reinterpret "marry" as "to cause someone to be married to another," which would lead to the conclusion that the man is authorized to conduct marriage ceremonies. Therefore, the essential characteristics of insight tasks involve directing attention away from the initial incorrect representations, allowing for the formation of novel mental representations.

Past studies using such insight problems have revealed that positive mood promotes performance on the tasks (e.g., Isen et al., 1987; Rowe et al., 2007), whereas negative mood impairs it (Subramaniam et al., 2009). One possible mechanism underlying these opposing influences of positive and negative moods is attention modulation. That is, positive mood serves to broaden the scope of attention (Fenske \& Eastwood, 2003; Fredrickson, 2004; Fredrickson \& Branigan, 2005; Gasper \& Clore, 2002; Rowe et al., 2007; Schmitz, De Rosa, \& Anderson, 2009), which should increase the likelihood that attention is diverted away from the initial incorrect representations and is redirected to competing representations during insight problem solving. In contrast, negative mood narrows one's attention (Bolte, Goschke, \& Kuhl, 2003; Fenske \& Eastwood, 2003). When people solve problems that require insight, this narrowed attention should cause them to focus on either the source of the negative emotion or the inappropriate initial representations. This narrowed attention should inhibit the process of redirecting attention away from the initial representation and block competing representations needed to come up with the solution, thus impairing insight problem solving.

Recent neuroimaging research provides evidence supporting the idea that subjective moods influence performance on insight problems through attention modulation. Subramaniam et al., (2009) compared brain activity during preparation for insight problems between participants with positive baseline mood states and participants with negative baseline mood states. Their results indicated that more positive feeling was associated with greater anterior cingulate (ACC) activity when preparing for insight problems. The ACC and adjacent medial prefrontal cortex (MPFC) have been implicated in conflict detection (e.g., Botvinick, Cohen, \& Carter, 2004; Nachev, Rees, Parton,
Kennard, \& Husain, 2005; Ridderinkhof, Ullsperger, Crone, $\&$ Nieuwenhuis, 2004). Therefore, it appears that positive mood states broaden attentional scope when preparing for insight problems, which leads to greater tendencies to search and find competing representations during problem solving, as reflected by the observation of increased activity in the ACC.

\section{The present study}

In the present study, we aimed to extend these previous findings by addressing the effects of brief presentations of emotional stimuli. In past studies, researchers have examined the effects of moods either by manipulating the participants' mood states before insight tasks (e.g., Isen, Johnson, Mertz, \& Robinson, 1985; Rowe et al., 2007) or by measuring individual baseline mood states in the participants (e.g., Subramaniam et al., 2009). However, recent research has revealed that the subjective feelings of emotion occur very soon after encountering emotional stimuli (e.g., within 500 ms; Rudrauf et al., 2009). Studies have also shown that attentional scope is modulated by even a brief presentation of emotional stimuli (e.g., 250400 ms; Baumann \& Kuhl, 2005; Dreisbach, 2006; Dreisbach \& Goschke, 2004; Fenske \& Eastwood, 2003). These findings suggest the possibility that cognitive flexibility is influenced not only by sustained mood states but also by transient emotional states induced by the simple viewing of emotional stimuli for short time periods.

To address this possibility, in the present study, we examined whether and how brief encounters with positive and negative images influence subsequent performance on insight tasks. In particular, we focused on the effects of emotional images on understanding of solutions to insight problems. In most behavioral studies on mood and cognitive flexibility, researchers have examined whether participants could solve insight tasks under certain moods (Alexander et al., 2007; Isen et al., 1987, 1985; Rowe et al., 2007; Subramaniam et al., 2009). In contrast, ACC and adjacent MPFC are related to insight tasks such that they are activated not only when people solve insight tasks on their own (Aziz-Zadeh, Kaplan, \& Iacoboni, 2009; Qiu et al., 2008), but also when they are provided with the solutions to insight tasks by other people (Luo \& Niki, 2003; Luo, Niki, \& Phillips, 2004; Mai, Luo, Wu, \& Luo, 2004; Qiu et al., 2006). This result suggests that ACC and MPFC are involved when people understand insight solutions that are shown to them. If brief encounters with positive and negative stimuli modulate attentional scope, such brief encounters should influence ACC and MPFC activity, which could facilitate or inhibit the understanding of insight solutions. 
In the present study, we employed riddles as problems that require insight because riddles involve important features of insight problems (Luo \& Niki, 2003; Luo et al., 2004), riddles induce mental impasse, and people have "Aha!" experiences when given or arriving alone at the solutions to riddles (Luo \& Knoblich, 2007; Mai et al., 2004). For each trial (see Fig. 1), participants were given a riddle (e.g., "What is so fragile that when you say its name you break it?") followed by a riddle-irrelevant emotional or neutral image (i.e., a modulator picture) for $660 \mathrm{~ms}$. In the control condition, participants were presented with a fixation cross instead of a modulator. After the modulator or the fixation cross disappeared, participants were presented with the solution to the riddle (e.g., "Silence") and were told to press a key immediately after they understood the solution. Given that past studies reported the attentional effects of positive stimuli even with a shorter presentation duration (e.g., 250-400 ms; Baumann \& Kuhl, 2005; Dreisbach, 2006; Dreisbach \& Goschke, 2004), we expected that viewing emotional images for $660 \mathrm{~ms}$ is long enough to change participants' moods and to influence subsequent understanding of the solutions to riddles. In Study 1 , we examined the effects of modulator valence on the time it took participants to report having understood the solutions. We did this in order to see whether the brief viewing of positive pictures facilitated understanding of the solutions to riddles and whether the brief viewing of negative pictures impaired understanding. In Study 2, we employed fMRI and examined the brain mechanisms by which the viewing of positive and negative pictures influences the understanding of insight problems.

\section{Study 1}

In Study 1, we addressed the effects of the brief viewing of positive and negative pictures on reaction times for understanding solutions to insight problems. If presentation of emotional images modulates attentional scope, participants should be able to understand the solutions more quickly following positive modulators as compared with neutral modulators or the fixation cross. In contrast, response times should be slower following negative modulator images compared to neutral modulators or the fixation cross.

We also tested participants' memory of emotional and neutral modulators. The results of past studies have revealed that both positive and negative stimuli are remembered better than neutral stimuli if they are matched in arousal levels (e.g., Dolcos \& Denkova, 2008; Hamann, Ely, Grafton, \& Kilts, 1999; Mather \& Sutherland, 2009). These findings suggest that arousal is more crucial than valence in the effects of emotion on memory. Therefore, we expected similar memory performances for positive and negative modulator pictures.

\section{Method}

Participants Thirty-one Japanese undergraduates at the University of Tokyo participated in the experiment (12
Fig. 1 A schematic representation of the experimental procedures in one trial for the positive condition

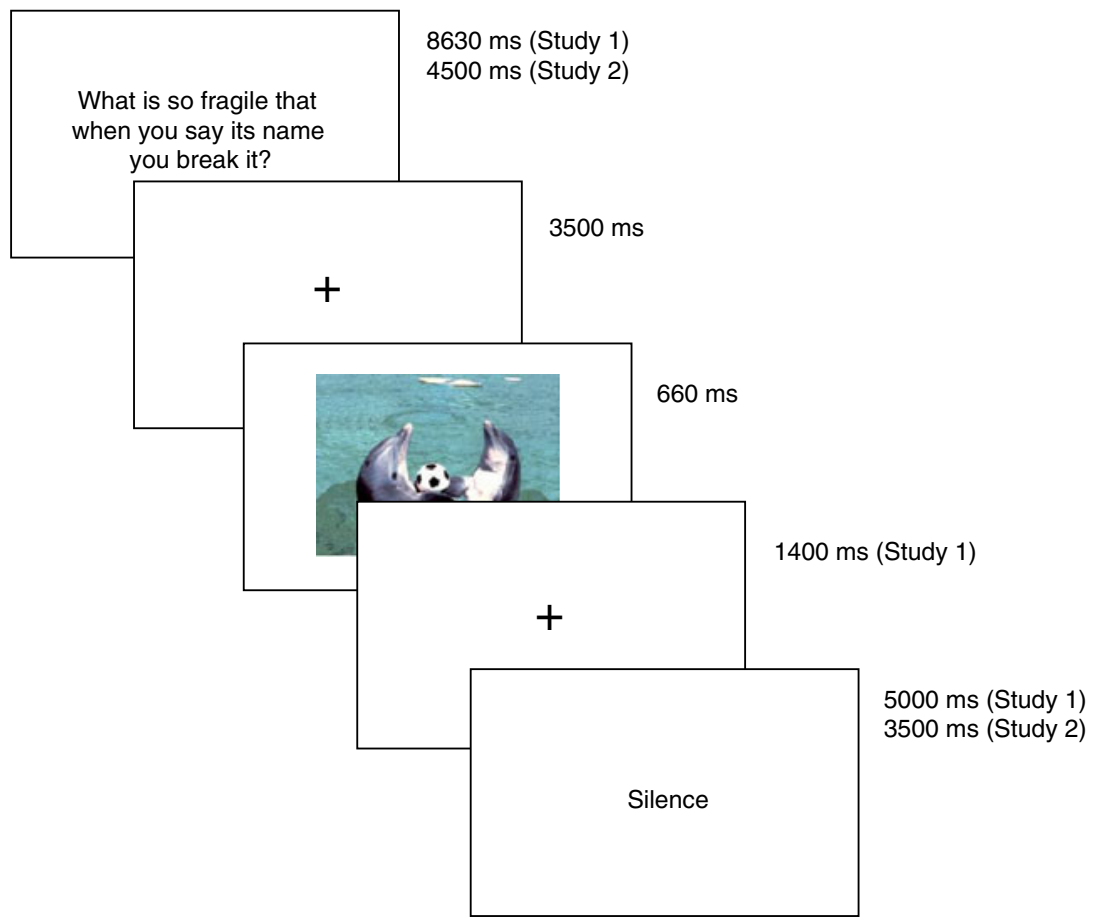


men; $M_{\text {age }}=20.2, S D=0.83$ ). Data from two participants were excluded because these participants did not finish the experiment.

Design The modulator type (positive, negative, neutral, or control) was manipulated as a within-participants factor. Eighteen trials were used for each condition.

Materials The materials used in Study 1 are described as follows.

Riddles Another sample of 13 Japanese undergraduate and graduate students were asked to solve a series of riddles obtained from a Japanese encyclopedia of riddles (Shibata, Tanikawa, \& Yagawa, 1984). Participants were allowed to think about each riddle for as long as they wanted. Seventytwo riddles, to which more than two-thirds of the pilot participants could not derive the correct solutions, were used in this study. These riddles were randomly assigned to each of the four conditions. The assignment of riddles to the conditions was counterbalanced across participants.

Modulator pictures Fifty-four pictures (18 positive, 18 negative, and 18 neutral pictures) were obtained from the International Affective Picture System (IAPS; Lang, Bradley, \& Cuthbert, 1997). Positive modulator pictures consisted of arousing and pleasant pictures (e.g., appealing animals, appetizing food, or sexually arousing scenes). Negative modulator pictures consisted of arousing and unpleasant pictures (e.g., snakes, guns, or aggressive behaviors). Neutral modulators consisted of low-arousing and neutral pictures (e.g., mundane activities or mushrooms). Positive, neutral and negative pictures were matched in terms of the number of pictures involving people.

Thirteen Japanese undergraduate and graduate students (four male) who did not participate in either Study 1 or 2 rated the valence (1 extremely negative; 9 extremely positive), the arousal level (1 low; 9 high), and the visual complexity (1 extremely simple; 9 extremely complex) of each picture. Positive pictures were rated as being significantly more positive than neutral and negative pictures, $t \mathrm{~s}$ $(24)=4.52,9.84 ; S E \mathrm{~s}=.22 ; r \mathrm{~s}=.68, .90 ; p \mathrm{~s}<.01$, and negative pictures were rated as being significantly more negative than neutral ones $\left(M_{\text {pos }}=5.9\right.$ vs. $M_{\text {neut }}=5.0$ vs. $\left.M_{\text {neg }}=3.8\right), t(24)=5.32, S E=.22, r=.74, p<.001$. The distance from neutral valence (valence score $=5$ ) did not differ between positive and negative pictures $(p>.15$; $M_{\text {neg }}=1.18$ vs. $\left.M_{\text {pos }}=0.96\right)$. Positive $(M=5.1)$ and negative $(M=5.2)$ pictures were also matched on arousal levels $(p>.80)$, and both picture types were rated as more arousing than neutral pictures $(M=4.2), t \mathrm{~s}(24)=4.02$, $4.61 ; S E \mathrm{~s}=.24 ; r s=.63, .69 ; p \mathrm{~s}<.01$. Furthermore, the complexity ratings did not significantly differ between positive $(M=3.59)$ and negative $(M=3.91 ; p>.05)$ pictures, though negative pictures were rated as more complex than neutral pictures $(M=3.49), t(24)=3.25$, $S E=.13, r=.55, p<.01$.

Procedure The 72 trials were randomly divided into three blocks of 24 trials. Each block included six positive, six negative, six neutral, and six control trials in a random order. As illustrated in Fig. 1, each trial started with the presentation of a riddle for $8,630 \mathrm{~ms}$ and was followed by a fixation cross for $3,500 \mathrm{~ms}$. A modulator picture was presented in the center of the screen for $660 \mathrm{~ms}$ in the positive, negative, and neutral conditions. Participants were told that the modulator was irrelevant to the riddle and were asked to look at the image passively. In the control condition, the fixation cross remained on the screen, and no picture was presented. After the modulator was replaced by the fixation cross for $1,400 \mathrm{~ms}$, a solution to the riddle was presented in the center of the screen for 5,000 ms. Participants were asked to press the "yes" key immediately after they understood the meaning of the solution and were asked to press the "no" key if they could not understand it. A blank screen was then presented for 5,500 ms until the next trial began.

After the riddle task, participants were asked to solve easy mathematical calculation problems for $3 \mathrm{~min}$, which was followed by a surprise recognition test for the modulator pictures. Participants were asked to indicate by a button press whether they remembered seeing the picture (i.e., old) or not (i.e., new). Eighteen nonstudied foils from each emotional category were included in this recognition task.

\section{Results}

Preliminary analyses revealed no significant effects of participants' sex in any dependent variables described in this section $(p s>.20)$. Participants' sex also did not modulate the effects of valence of modulator pictures $(p s>.10)$.

Effects of the valence of modulators on understanding of the solutions to riddles For most of the trials (86\%), participants reported that they understood the meaning of the solution. Therefore, for each participant, trials in which the participant could not understand the solutions were discarded from the analysis in this section. The number of trials in which participants could not understand the solutions did not differ significantly across conditions $(p>.20)$. Outliers for response times to the solution were identified using Tukey's (1977) criterion; five individual trials producing extreme outliers, which were three times higher than the third quartile of the interquartile range (i.e., the hinge-spread), were excluded. 
A one-way ANOVA on the reaction times to report understanding the solutions revealed a significant main effect of the modulator type, $F(3,87)=6.16, p<.01$, $\eta_{\mathrm{p}}{ }^{2}=.09$. Participants were faster to report that they understood the solutions in the positive condition than in the neutral or control conditions (see Fig. 2), $F(1,87)=$ $6.53, p<.05$. In contrast, participants showed slower reaction times in the negative condition than in the neutral or control conditions, $F(1,87)=4.84, p<.05$. These results suggest that the brief viewing of positive and negative images has opposing effects on understanding of the solutions to riddles.

To address the effects of the visual complexity of modulator pictures, pictures were categorized into complex and simple picture sets, using the median of the pilot complexity ratings (see the Materials section) in each valence category. A 3 (modulator valence: positive, negative, neutral) X 2 (modulator complexity: simple, complex) ANOVA on the reaction times to solutions confirmed a significant effect of modulator valence, $F(2,58)=9.44$, $\eta_{\mathrm{p}}{ }^{2}=.25, p<.05$, with no other significant results $(p s>.20)$. The effects of valence were significant both in complex, $F(2,58)=3.57, \eta_{\mathrm{p}}{ }^{2}=.11, p<.05$, and in simple picture sets, $F(2,58)=7.47, \eta_{\mathrm{p}}{ }^{2}=.20, p<.01$. Thus, it appears that the effects of modulator valence cannot be attributed to picture complexity.

Memory of modulator pictures A one-way ANOVA on the corrected recognition measure (i.e., hits minus false alarm rates) yielded a significant effect of the modulator valence, $F(2,58)=29.18, p<.001, \eta_{\mathrm{p}}{ }^{2}=.50$. Participants recognized positive and negative modulators more accu-

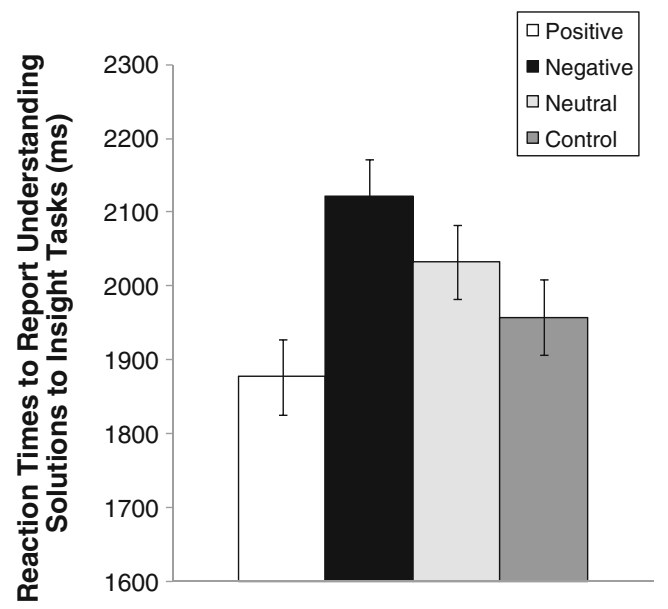

Fig. 2 Mean response times to report having understood the solutions to riddles in Study 1 (error bars indicate standard errors of the means). Participants were faster to report that they understood the riddle solutions following positive images. In contrast, viewing of negative images led to slower response times to report understanding the solutions. rately than neutral modulators (see Table 1$), t \mathrm{~s}(58)=6.16$, 6.99; $S E \mathrm{~s}=.03 ; r=.63,68 ; p \mathrm{~s}<.001$. However, there were no significant differences in recognition of positive and negative pictures $(p>.60)$. We also examined the effects of picture complexity based on the median split. A 3 (valence) X 2 (complexity) ANOVA on the hit rates confirmed a significant effect of valence, $F(2,58)=$ 21.86, $\eta_{p}^{2}=.43, p<.01$, with no other significant effects $(p \mathrm{~s}>.25)$. Thus, as in the reaction time results, the complexity of pictures did not modulate the effects of valence on memory.

To examine the effects of memory for the modulator pictures on the solution reaction times, a 3 (modulator valence) X 2 (memory for modulators: remembered, forgotten) ANOVA was performed on the reaction times to report understanding the solutions. Replicating the results reported in the previous section, this ANOVA confirmed a significant effect of valence, $F(2,58)=6.32, \eta_{\mathrm{p}}{ }^{2}=.18$, $p<.01$. Neither the main effect of memory nor the interaction between memory and valence was significant $(p s>.70)$. Thus, positive modulator pictures produced faster reaction times to report understanding the solutions, but negative modulator pictures increased the reaction times, regardless of whether participants remembered $\left(M_{\text {pos }}=2,074 \mathrm{~ms}, M_{\text {neut }}=2,245 \mathrm{~ms}\right.$, vs. $M_{\text {neg }}=$ 2,397 $\mathrm{ms}$ ) or forgot those modulator pictures in the subsequent memory test $\left(M_{\text {pos }}=2,047 \mathrm{~ms}, M_{\text {neut }}=\right.$ $2,242 \mathrm{~ms}$, vs. $\left.M_{\text {neg }}=2,326 \mathrm{~ms}\right)$.

\section{Discussion}

The results of past studies have revealed that performance on insight tasks is facilitated by positive mood states but is impaired by negative mood states (e.g., Subramaniam et al., 2009). The results of Study 1 extend these previous findings to suggest that brief viewings of positive and negative images also have opposing effects on understanding of the solutions to insight-like problems. In addition, the effects of modulator pictures on the understanding of riddle solutions did not differ depending on whether the modulator pictures were subsequently remembered or not. These results suggest that the effects of emotional pictures on understanding solutions to insight problems do not depend on similar mechanisms as do the effects of emotional pictures on memory.

\section{Study 2}

In Study 2, we employed fMRI to examine the brain mechanisms by which brief presentations of positive and negative stimuli influence understanding of the solutions to insight problems. Recent neuroimaging studies have 
Table 1 Results from the recognition memory tests of the modulator pictures (standard errors in parentheses)

\begin{tabular}{|c|c|c|c|c|c|c|}
\hline & \multicolumn{3}{|c|}{ Hit - False alarm } & \multicolumn{3}{|l|}{ Hit } \\
\hline & Positive & Negative & Neutral & Positive & Negative & Neutral \\
\hline \multirow[t]{2}{*}{ Study 1} & .65 & .63 & .44 & .66 & .70 & .49 \\
\hline & $(0.03)$ & $(0.03)$ & $(0.03)$ & $(0.02)$ & $(0.02)$ & $(0.02)$ \\
\hline \multirow[t]{2}{*}{ Study 2} & .43 & .43 & .23 & .48 & .50 & .28 \\
\hline & $(0.05)$ & $(0.05)$ & $(0.05)$ & $(0.05)$ & $(0.05)$ & $(0.05)$ \\
\hline
\end{tabular}

revealed several brain regions that are involved in processing of insight problems. These regions include the superior temporal gyrus (Bowden et al., 2005; Jung-Beeman et al., 2004), the ACC and adjacent MPFC (Dietrich \& Kanso, 2010; Kunosis et al., 2006; Luo et al., 2004; Subramaniam et al., 2009), and the angular gyrus/temporo-parietal junction (Aziz-Zadeh et al., 2009; Bechtereva, Korotkov, Pakhomov, Roudas, Starchenko, \& Medvedev, 2004; Fink et al., 2009; Jung et al., 2010; Luo et al., 2004; Mai et al., 2004). In Study 2, we addressed whether the brief viewing of emotional images affected activity in these regions during processing of the solutions to riddles.

\section{Method}

Participants Fourteen Japanese undergraduate and graduate students at the University of Tsukuba participated in the experiment $\left(10 \mathrm{men} ; M_{\text {age }}=21.71, S D=2.46\right)$. Each participant gave informed consent in accordance with the MRI ethics committee of the National Institute of Advanced Industrial Science and Technology. Prospective participants were excluded if they had any medical, neurological, or psychiatric illness.

Design The experimental design was identical to that in Study 1, except that we added two filler trials at the beginning of the scanning session to familiarize participants with the task in the MRI scanner. The procedures of the filler trials were the same as those of the trials in the control condition.

Materials and behavioral procedure Before entering the scanner, participants were presented with 121 Japanese riddles obtained from Shibata, Tanikawa, and Yagawa (1984). For each riddle, participants were asked to indicate whether or not they knew the correct solution. During the fMRI scan, 74 riddles to which the participant did not know the correct solution were used. Among those 74 riddles, two riddles were used in the filler trials. The remaining 72 riddles were randomly assigned to each of the four conditions for each participant. Participants were also given a practice session outside of the scanner. None of the materials used in the practice session was presented during scanning. After participants entered the scanner, an initial T1-weighted sagittal localizer and a T1-weighted axial image were acquired. This procedure was followed by an fMRI scanning session.

The task procedures were similar to those in Study 1, with several modifications in the stimulus presentation times. First, we reduced the duration of the riddle presentation because participants had already seen each riddle before they entered the scanner. We also reduced the duration of the solution presentation according to the reaction time data from Study 1. In addition, we increased the duration of the interstimulus intervals (ISIs) between the distractors and the solutions and introduced a temporal jitter to the intertrial intervals (ITIs) to separate the BOLD signals between the events and between trials. During the task, participants first saw a riddle for $4,500 \mathrm{~ms}$ (see Fig. 1), followed by a fixation cross for 3,500 ms. They were asked to press the "yes" or the "no" key during the presentation of the fixation cross to indicate whether they had come up with a possible solution to the riddle. ${ }^{1}$ After the presentation of the fixation cross, a modulator picture appeared for $660 \mathrm{~ms}$ and was followed by the fixation cross for $2,000 \mathrm{~ms}$. In the control condition, the fixation cross remained instead of the modulators. Finally, the correct solution to the riddle was presented for 3,500 ms. Participants were asked to indicate whether they could understand the solution by pressing keys.

After exiting the scanner (approx 5 min), a surprise recognition test on the modulator pictures was given. The procedures for the recognition test were identical to those in Study 1. Finally, participants rated emotional and neutral pictures they saw during the riddle task in terms of valence (1 negative; 7 positive) and arousal (1 low; 7 high).

fMRI data acquisition and preprocessing All scanning was performed in a 3.0-T MRI Scanner (GE $3 \mathrm{~T}$ Signa)

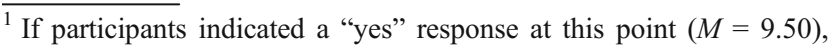
they were asked to recall what they had in mind as a solution for the corresponding riddle after the experiment. No participants reported that they had come up with the correct solution to any items at this point.
} 
equipped with echo-planar imaging (EPI) capability using the standard head coil for radiofrequency transmission and signal reception. Twenty-seven axial slices (4-mm thick with a $0.2-\mathrm{mm}$ gap, interleaved) were prescribed to cover the entire brain. A T2*-weighted gradient echo EPI was employed. The imaging parameters were as follows: TR $=$ $2 \mathrm{sec}, \mathrm{TE}=30 \mathrm{~ms}, \mathrm{FA}=75, \mathrm{FOV}=20 \mathrm{~cm} \times 20 \mathrm{~cm}(64 \times$ $64 \mathrm{mesh}$ ). To avoid head movement, participants wore a neck brace and were asked not to talk or move during scanning. Foam padding was also used to limit head movement. Each participant's data were individually preprocessed by SPM5 (Wellcome Trust Center for Neuroimaging). In the preprocessing analysis, images were corrected for slice-timing and motion and were then spatially normalized to the Montreal Neurological Institute (MNI) template and spatially smoothed using a Gaussian kernel of 8-mm FWHM.

fMRI whole-brain analysis Task-dependent changes in BOLD signals were modeled with a regressor for each event (riddle question, modulator, and solution) of each type of modulator condition. Solutions which the participant indicated, by pressing the "no" key, that he or she could not understand were modeled separately. Because only a few trials fell into this category (see the Behavioral Results section below), we did not perform any statistical tests for these items. The regressors were convolved with the canonical hemodynamic response function provided by SPM. A high-pass filter (cutoff period $=128 \mathrm{~s}$ ) was applied to remove low-frequency artifacts from the data. The effects of each event type were estimated using a fixed-effects model, and the results of the fixed-effects analysis were entered into a random-effects analysis. The threshold was set at $p<.05$, familywise error corrected (FWE) at cluster level with a height threshold of $Z>2.3$ (Worsley, 2001). Locations reported by SPM were converted into Talairach coordinates by the MNI-to-Talairach transformation algorithm (Lancaster et al., 2007). These coordinates were used to determine the nearest gray matter using the Talairach Daemon program Version 2.4.2 (Lancaster et al., 2000).

Regions of interest analysis To address the effects of memory for modulator pictures on brain activity during solution processing, we constructed a new general linear model (GLM) design file in which we separately modeled remembered modulators, forgotten modulators, solutions following remembered modulators, and solutions following forgotten modulators for each valence category. Using the MarsBar toolbox (Brett, Anton, Valabregue, \& Poline, 2002), regions of interest (ROI) analyses were performed on brain regions where we found significant effects of the modulator types in the previous whole-brain analysis.
Correlation analyses between brain activity and reaction times to solutions To identify the role of the brain regions revealed in the previous whole-brain analysis, we examined the relationship between brain activity and reaction times to report understanding the solutions. As a first step, we constructed another GLM design file in which each event (i.e., question, modulator, and solution) for each individual trial was coded as a unique covariate. To reduce the confounding effects of the global signal change, we calculated the global mean signal level over all brain voxels for each time point and used the global mean signal level as a covariate. The least squares solution of the GLM yielded a beta value for each event on each trial for each individual participant. For the solution in each trial for each participant, percent signal changes were obtained from regions in which we found significant effects of the emotional modulator in the previous whole-brain analysis. These percent signal changes were used to examine the effects of brain activity on the reaction times.

Because the data had a hierarchical structure in which each trial was nested in each participant, we employed a hierarchical multilevel regression analysis (HLM: Raudenbush \& Bryk, 2002). The independent variables were the percent signal changes in the brain regions during the solution for each trial. The dependent variable was the reaction time to report understanding the solution during the trial. To control for the possible confounding effects of the modulator valence, the modulator type condition (i.e., control, positive, negative, and neutral) for each trial was also included as an additional independent variable. This analysis allowed us to examine how strongly intraindividual differences in brain activity (i.e., trial-by-trial variance) were related to intra-individual differences in reaction times to understand the solutions while dealing with the multilevel data appropriately and controlling the overall effects of the modulator valence.

\section{Behavioral results}

Preliminary analyses revealed no significant effects of participants' sex $(p s>.40)$ and no significant interactions between sex and modulator valence condition $(p s>.15)$ in any behavioral measures reported in this section (i.e., reaction times to the solutions, ratings of modulator pictures, and memory for modulator pictures).

Ratings of modulator pictures Participants rated positive pictures more positively than neutral and negative pictures, $t \mathrm{~s}(26)=6.46,13.13 ; S E \mathrm{~s}=.21 ; r \mathrm{~s}=.79, .93 ; p \mathrm{~s}<.001 ;$ and negative pictures more negatively than neutral pictures $\left(M_{\text {pos }}=5.29\right.$ vs. $M_{\text {neut }}=3.91$ vs. $\left.M_{\text {neg }}=2.50\right), t(26)=6.67$, 
$S E=.21, r=.79, p<.001$. The distance from neutral valence (valence score $=4$ ) did not differ between positive and negative pictures $(p>.30)$. Positive $(M=4.09)$ and negative $(M=3.85)$ pictures were higher in arousal ratings than neutral ones $(M=2.31), t \mathrm{~s}(26)=5.03,5.81 ; S E \mathrm{~s}=.31$; $r \mathrm{~s}=.70, .75 ; p \mathrm{~s}<.001$, whereas the arousal ratings did not differ between positive and negative pictures $(p>.70)$.

Effects of the valence of modulators on understanding of the solutions to riddles As in Study 1, participants understood the solutions in the majority of the trials (71\%). The number of trials in which participants could not understand the solutions did not differ significantly across conditions $(p>.65)$.

After trials in which participants could not understand the solutions were discarded, we examined whether participants showed different reaction times to report understanding the solutions depending on the type of modulators presented. Outliers for solution response times were identified using the same criteria as in Study 1. A oneway ANOVA revealed a significant effect of modulator type, $F(3,39)=3.97, \eta_{\mathrm{p}}{ }^{2}=.23, p<.05$. Because the number of participants was smaller in this study than in Study 1, subsequent analyses revealed a significant difference only between the positive and negative conditions, $t(39)=2.80, S E=71, p<.05$. However, the overall patterns were consistent with Study 1. In other words, participants were slower to report that they understood the solution in the negative condition $(M=2,704 \mathrm{~ms})$ than in the neutral or control conditions ( $M \mathrm{~s}=2,557 \mathrm{~ms} ; 2,664 \mathrm{~ms}$, respectively), in which participants were slower compared as compared with the positive condition ( $M=2505 \mathrm{~ms})$.

An additional analysis was performed to address the effects of the visual complexity of pictures. Using the pilot complexity ratings, pictures were split by the median complexity score in each valence category. A 3 (modulator valence: positive, negative, neutral) X 2 (modulator complexity: simple, complex) ANOVA on the reaction times to report understanding the solutions confirmed a significant effect of modulator valence, $F(2,26)=5.69, \eta_{\mathrm{p}}{ }^{2}=.30, p<.05$, with no other significant results $(p s>.20)$. Thus, as in Study 1, it appears that the effects of modulator valence cannot be attributed to picture complexity.

Memory of emotional modulators A one-way ANOVA on the corrected recognition measure yielded a significant effect for the modulator valence, $F(2,26)=8.72, p<.001$, $\eta_{\mathrm{p}}{ }^{2}=.40$. Participants showed more accurate memory for the positive and negative modulators than for the neutral modulators (Table 1), $t \mathrm{~s}(26)=3.58,3.65 ; S E \mathrm{~s}=.05$; $r \mathrm{~s}=.57, .58 ; p \mathrm{~s}<.001$. There were no significant differences between the positive and negative modulators $(p>.90)$. To address the effects of the picture complexity, a
3 (modulator valence) X 2 (modulator complexity) ANOVA was performed on the hit rates based on the median split of the pilot complexity ratings. This ANOVA confirmed a significant effect of modulator valence, $F(2,26)=11.53$, $\eta_{\mathrm{p}}{ }^{2}=.43, p<.01$, with no other significant effects $(p \mathrm{~s}>.15)$. Thus, the complexity did not modulate the effects of valence on memory.

To examine the relationships between memory for the modulator pictures and the reaction times to the solutions, we performed a 3 (modulator valence) $\mathrm{X} 2$ (modulator memory: remembered, forgotten) ANOVA on the reaction times to report understanding the solutions. This ANOVA confirmed a significant effect of the modulator valence, $F(2,24)=4.41, p<.05, \eta_{\mathrm{p}}{ }^{2}=.24$, with no other significant effects $(p \mathrm{~s}>.25)$. Therefore, as in Study 1, the memory for modulator pictures did not influence the effects of emotional pictures on the time to understand the solutions.

Functional MRI results

Effects of emotional modulators on brain activity during solution processing A whole-brain analysis examining brain activity during processing of riddle solutions revealed greater activity in the right angular gyrus (Fig. 3a) and in the bilateral MPFC (Fig. 3b-c) following positive pictures as compared with the fixation cross (Table 2). In contrast, negative and neutral modulator pictures did not produce significant differences from the fixation cross. Furthermore, when we contrasted the positive and the negative conditions, the solutions to riddles produced greater activity in the right angular gyrus (Fig. 3d) and in right MPFC (Fig. 3e-f) after positive modulator pictures than negative ones (Table 2). A reversed contrast did not find any significant results.

To address the effects of memory for modulator pictures on brain activity while processing solutions, we performed ROI analyses on 3-mm spheres surrounding the peak voxel of the activation clusters where we found significant effects of emotional modulators in the previous analysis: the right angular gyrus [50, -68, 34] and MPFC [10, 28, 44]. The results indicated that positive modulators produced greater right angular gyrus activity than the fixation cross, regardless of whether those modulators were remembered, $t=3.06, p<.05$, or forgotten in the subsequent recognition test, $t=1.81, p<.05$. Positive pictures also produced greater activity in MPFC regardless of the memory performance (remembered: $t=1.61, p<.07$; forgotten: $t=2.01, p<.05$ ), although the remembered items showed only marginal effects. In fact, there were no significant differences in right angular gyrus and MPFC activities between the remembered positive and forgotten positive images while participants processed solutions $(t \mathrm{~s}=-0.57$ 

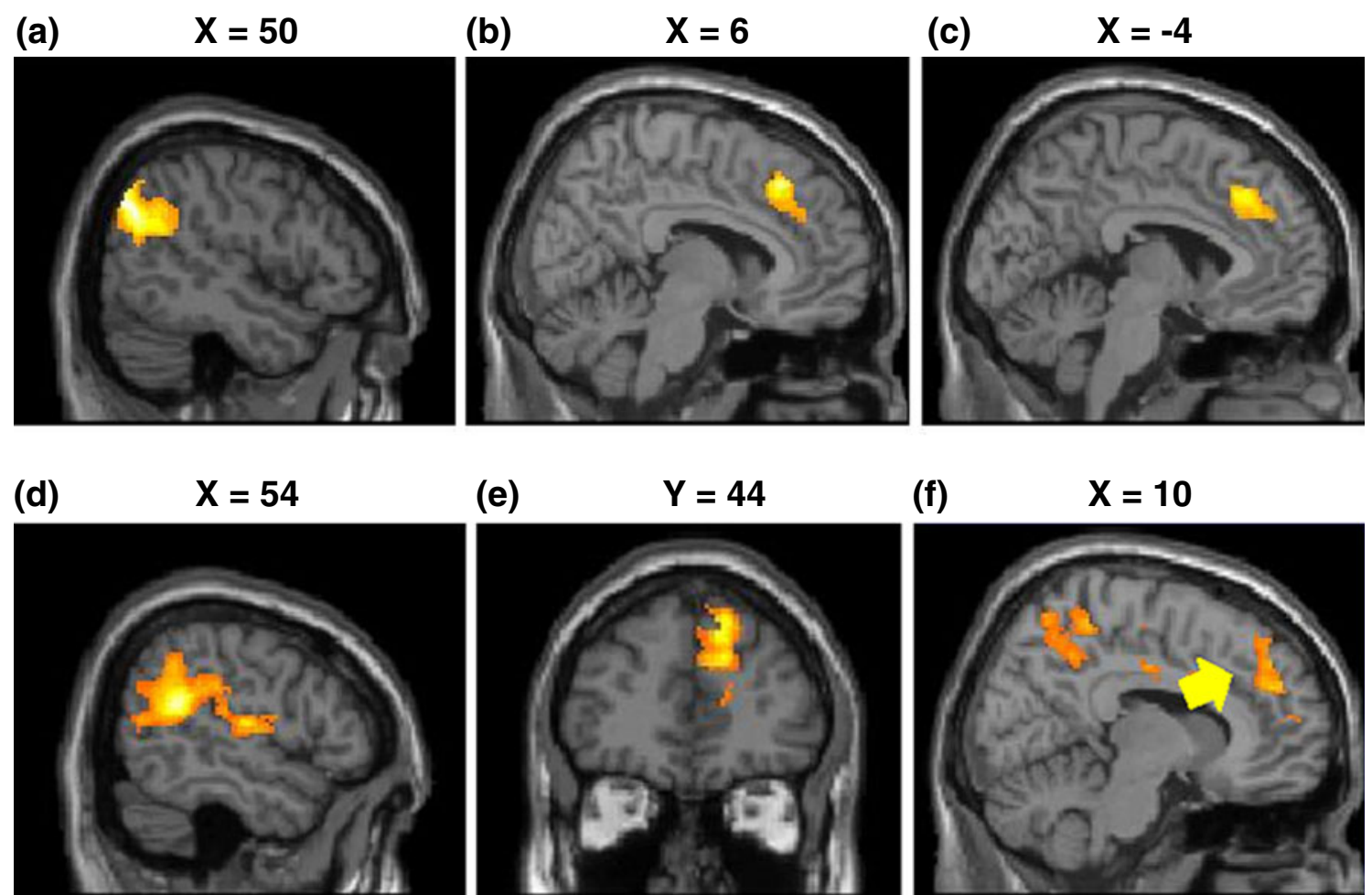

(e)

(f) $\quad X=10$
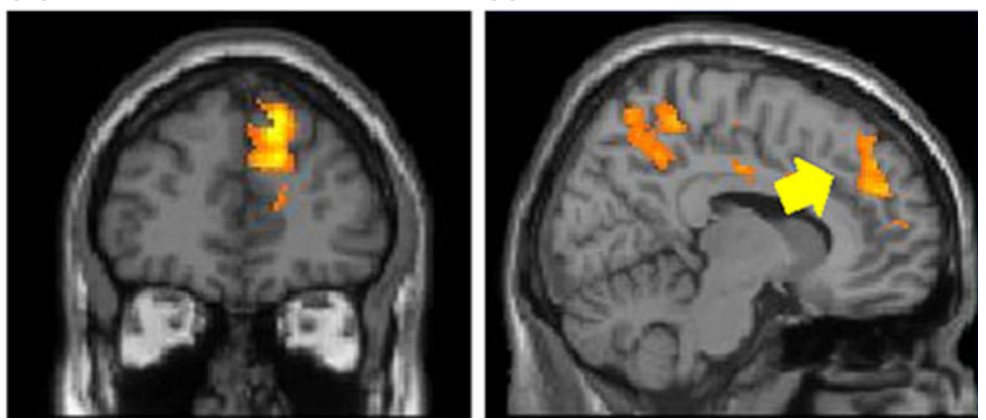

Fig. 3 Compared with the control condition, positive modulator pictures produced greater activity in (a) right angular gyrus, (b) right MPFC, and (c) left MPFC during processing solutions. Similar regions were also revealed when we compared positive modulator

and 0.07 respectively). These results are consistent with the behavioral results that memory for modulator pictures did not influence the effects of emotional pictures on understanding of the solutions to insight problems. ${ }^{2}$

Correlational analyses between brain activity and the reaction times to solutions HLM analyses were employed to address the roles of the right angular gyrus and MPFC in understanding the solutions to riddles. The dependent variable was the reaction time to report understanding the solutions for each trial in each participant. The independent variables were the percent signal changes from 3-mm spheres around the peak voxels in the right angular gyrus and MPFC for the solution in each individual trial. To control for the effects of the modulator valence, which was revealed to influence both the reaction times (i.e., the dependent variable) and the activity of the regions included as independent variables, the modulator type condition (i.e.,

\footnotetext{
${ }^{2}$ To address the effects of the picture complexity, post-hoc ROI analyses were performed on the activity in the 3-mm spheres around the peak voxels in the right angular gyrus and MPFC, using the pilot complexity ratings of each modulator picture. The results indicated that the picture complexity did not influence the activity of the right angular gyrus and MPFC ( $p$ s $>.15)$. The picture complexity also did not significantly modulate the effects of modulator valence on activity in those regions $(p s>.15)$.
}

pictures with negative ones: As compared with negative pictures, positive modulator pictures produced greater activity in (d) right angular gyrus and $(\mathbf{e}, \mathbf{f})$ right MPFC

control, positive, negative, and neutral) in each trial was also included as an additional covariate. The results indicated that the activity in the right angular gyrus predicted faster reaction times to report understanding the riddle solutions (unstandarized $\beta=-91$ ), $F(1,720)=4.04$, $p<.01$. The effects of MPFC activity were not significant $(p>.40)$. Thus, it appears that the positive modulator pictures induced greater activity in the right angular gyrus, which predicted faster understanding of the solutions to riddles.

\section{Discussion}

In Study 2, we addressed the brain mechanisms by which brief viewings of emotional pictures influence the understanding of solutions to insight-like problems. As compared with the control condition, viewing of positive pictures enhanced activity in the right angular gyrus and the MPFC while participants processed the solutions to riddles. Similar results were obtained when the positive condition was contrasted to the negative condition. These results suggest that brief presentation of emotional stimuli influences subsequent understanding of the solutions to insight problems by modulating the activity in the angular gyrus and the MPFC. Consistent with this idea, the greater the right angular gyrus was activated, the more quickly 
Table 2 Brain areas showing differential activity by the valence of emotional modulators while processing the solutions to riddles (MNI coordinates)

\begin{tabular}{|c|c|c|c|c|c|c|}
\hline & $\mathrm{H}$ & $\mathrm{BA}$ & $\mathrm{x}$ & $\mathrm{y}$ & $\mathrm{z}$ & $\mathrm{K}$ \\
\hline \multicolumn{7}{|l|}{ Positive $>$ Control } \\
\hline \multirow[t]{2}{*}{ Angular Gyrus } & $\mathrm{R}$ & 39 & 50 & -68 & 34 & 993 \\
\hline & $\mathrm{R}$ & 39 & 46 & -70 & 42 & \\
\hline Middle Temporal Gyrus & $\mathrm{R}$ & 39 & 42 & -64 & 22 & \\
\hline \multirow[t]{3}{*}{ MPFC } & $\mathrm{R}$ & 8 & 10 & 28 & 44 & 974 \\
\hline & $\mathrm{R}$ & 8 & 14 & 40 & 34 & \\
\hline & $\mathrm{L}$ & 8 & 0 & 32 & 42 & \\
\hline \multicolumn{7}{|l|}{ Positive $>$ Negative } \\
\hline \multirow[t]{2}{*}{ Angular Gyrus } & $\mathrm{R}$ & 39 & 64 & -32 & 24 & 11786 \\
\hline & $\mathrm{R}$ & 39 & 46 & -34 & 30 & \\
\hline Superior Temporal Gyrus & $\mathrm{R}$ & 19 & 52 & -48 & 20 & \\
\hline MPFC & $\mathrm{R}$ & 9 & 14 & 50 & 26 & \\
\hline Middle Cingulate Gyrus & $\mathrm{L}$ & 24 & -6 & -12 & 34 & \\
\hline \multirow[t]{2}{*}{ Supramarginal Gyrus } & $\mathrm{L}$ & 40 & -56 & -38 & 40 & 673 \\
\hline & $\mathrm{L}$ & 40 & -54 & -48 & 48 & \\
\hline Superior Temporal Gyrus & $\mathrm{L}$ & 22 & -64 & -52 & 26 & \\
\hline \multicolumn{7}{|l|}{ Negative $>$ Control } \\
\hline \multicolumn{7}{|l|}{ No significant results } \\
\hline \multicolumn{7}{|l|}{ Negative $>$ Positive } \\
\hline \multicolumn{7}{|l|}{ No significant results } \\
\hline \multicolumn{7}{|l|}{ Neutral $>$ Control } \\
\hline No significant results & & & & & & \\
\hline
\end{tabular}

participants reported that they understood the solutions to the riddles, though the activity in the MPFC did not have significant effects on the reaction times.

\section{General discussion}

In the present study, we addressed the effects of brief viewings of positive and negative images on subsequent understanding of the solutions to insight problems. As shown in past studies (e.g., Dolcos \& Denkova, 2008; Mather \& Sutherland, 2009), participants remembered both positive and negative images equally better than neutral images. Despite the similar memory enhancement effects, presentation of positive and negative pictures had different impacts on subsequent understanding of the solutions to problems requiring insight. That is, brief viewing of positive pictures led to faster response times to report understanding the solutions, whereas viewing negative pictures for the same duration increased the response times. The results of Study 2 revealed that as compared with the control condition, the presentation of positive images increased activity in the right angular gyrus and the MPFC during processing of the solutions. Furthermore, the activity of the right angular gyrus was associated with faster reaction times to report understanding the solutions to insight problems. In contrast, negative modulator pictures did not produce similar activity during processing of the solutions. In fact, positive modulator pictures produced greater activity in MPFC and the right angular gyrus, even when contrasted with negative pictures. These results suggest that the brief viewing of positive and negative stimuli has different influences on the understanding of the solutions to insight problems.

Past studies have shown that cognitive flexibility is influenced by people's sustained mood states (e.g., Isen \& Daubman, 1984; Isen et al., 1987, 1985; Rowe et al., 2007; Subramaniam et al., 2009). Whereas in most of the previous studies researchers targeted the effects of sustained mood states that lasted for at least several minutes, in the present study, we employed a brief presentation of emotional stimuli (i.e., $660 \mathrm{~ms}$ ). Furthermore, the four modulator type conditions were presented randomly mixed across trials instead of being presented as blocks of negative, positive, neutral, and control trials. Despite these subtle manipulations, as compared with past studies, we observed reliable effects of modulator valence both on the behavioral reaction times and on brain activity during the processing of the solutions to insight-like problems. In fact, brief presentation of positive images produced greater activity in the MPFC, which has been implicated in the effects of sustained mood states on insight task performance. That is, Subramaniam et al. (2009) revealed greater ACC and adjacent MPFC activity while preparing for insight problem solving in people with positive mood as compared with those in a negative mood. Subramaniam et al. also reported that when preparing for new problems, positive moods increased activity in the right angular gyrus - the area in which we found the enhanced activity following positive modulator pictures. Thus, it appears that emotional states can be changed by simply viewing emotional pictures for a short time period, which further influences cognitive flexibility by modulating activity in brain regions similar to those that are modulated by long-lasting mood states.

Mechanisms by which the brief viewing of emotional stimuli affects understanding of insight solutions

Facilitative effects of positive stimuli As described above, the MPFC and the right angular gyrus showed increased activity following the viewing of positive images as compared with the fixation cross or negative images. The MPFC and adjacent ACC have been implicated in conflict detection (Botvinick et al., 2004; Ridderinkhof et al., 2004). For example, these regions are activated during the Stroop task (MacLeod \& MacDonald, 2000), in which people must detect conflicts between the correct responses and the 
responses being overridden. These areas are also activated when individuals detect conflicts between action plans (Nachev et al., 2005).

In contrast, the angular gyrus has been implicated in directing attention to behaviorally relevant stimuli that are outside the focus of attention (Corbetta, Kincade, \& Shulman, 2002; Luo, Niki, \& Knoblich, 2006). For example, the angular gyrus shows increased activity when directing attention to target stimuli presented at an unattended location (Indovina \& Macaluso, 2007). Transcranial magnetic stimulation administered in this region also disrupted the shift of attention from one visual cue to another visual stimulus (Chambers, Payne, Stokes, \& Mattingley, 2004). In addition, the angular gyrus becomes activated when shifting attention to mentally generated stimuli, such as retrieved episodic memories (see Cabeza, Ciaramelli, Olson, \& Moscovitch, 2008, for a review).

These findings from past studies provide clues about the roles of MPFC and the angular gyrus in understanding the solutions to insight problems. That is, the MPFC appears to help recognize competing representations (e.g., "marrying" can have several different meanings). In contrast, the angular gyrus might help redirect attention away from the initial inappropriate representations (e.g., "marrying" meaning "taking someone in marriage") to the correct representation (e.g., "marrying" meaning "binding others together in marriage"), allowing one to understand the provided solutions (e.g., the man is authorized to conduct marriage ceremonies). Because we observed an increased activity in these two regions in the positive condition, we suggest that the brief presentation of positive pictures broadens the attentional scope, which increases (a) the likelihood that people recognize competing representations, reflected by an increased activity in MPFC, and (b) the likelihood that people draw attention away from initial inappropriate mental representations to the correct representations, which is implemented by the angular gyrus.

Inhibitory effects of negative stimuli In contrast with the facilitative effects of positive stimuli, we observed that viewing of negative stimuli delayed reaction times to report understanding the solutions. However, there were no significant differences in brain activity between the negative and the control conditions during processing of the solutions. The results of previous studies also reported that the effects of negative mood on cognitive flexibility are relatively weak as compared with the effects of positive mood (see Baas et al., 2008; Davis, 2009, for reviews). This weak nature of the inhibitory effects of negative mood might be a reason for the lack of significant differences in brain activity between the negative and the control conditions. Further studies should clarify how viewing of negative stimuli influences subsequent understanding of insight solutions.

Valence versus arousal effects

Results from past studies in which emotional stimuli were presented briefly as distractors for a subsequent cognitive task have revealed that brief presentations of emotionally arousing stimuli interfere with subsequent cognitive processing. For example, the rapid presentation of emotional stimuli impairs perceptual identification of subsequent stimuli (Anderson, 2005; Arnell, Killman, \& Fijavz, 2007; Ihssen \& Keil, 2009; Most, Chun, Widders, \& Zald, 2005). Similar interference effects have been observed in a wide variety of tasks, such as lexical judgments (Calvo \& Castillo, 2005; Ihssen, Heim, \& Keil, 2007), the Stroop task (McKenna \& Sharma, 1995), digit-parity judgments (Aquino \& Arnell, 2007), the n-back task (Kensinger \& Corkin, 2003), short-term memory retrieval (Dolcos, Kragel, Wang, \& McCarthy, 2006; Dolcos \& McCarthy, 2006), and mathematical calculations (Schimmack, 2005). Although some of these studies used only negative stimuli, studies that employed positive and negative stimuli observed effects of arousal rather than effects of valence (Ihssen et al., 2007; Mather, Mitchell, Raye, Novak, Greene, \& Johnson, 2006; Schimmack, 2005).

An obvious question raised by these past findings is whether valence is crucial in explaining the differential impact of positive and negative images in the present study. In fact, negative stimuli are usually higher in subjective arousal level than are positive stimuli (Lang et al., 1997). Although positive and negative pictures did not differ in the arousal ratings both in the pilot rating study and in Study 2, the procedures of the picture rating task were different from those in the riddle task. For example, participants were allowed to view each picture as long as they wanted in the picture rating task. In contrast, during the riddle task, participants viewed pictures for only $660 \mathrm{~ms}$, and they were intermixed with riddles and solutions. Therefore, it is possible that the positive and negative images evoked different levels of arousal during the riddle task and that our results are due to differences in the arousal levels between the positive and negative modulator pictures instead of valence.

However, in both Studies 1 and 2, we observed that the positive and negative modulator pictures were both remembered equally better than the neutral pictures. Emotional memory enhancement has been attributed to arousal (Mather \& Sutherland, 2009), and positive and negative stimuli are equally remembered when they are matched in arousal (e.g., Hamann et al., 1999). Activity in the amygdala, which is a key region for emotional memory 
enhancement (Canli, Zhao, Brewer, Gabrieri, \& Cahill, 2000; Dolcos, LaBar, \& Cabeza, 2004; Hamann et al., 1999), is also known to be modulated by the arousal level of stimuli rather than valence (i.e., positive or negative; Anderson et al., 2003; Kensinger \& Corkin, 2004). Given the similar memory performance between the positive and negative stimuli we observed, it is unlikely that the arousal level was substantially different between the positive and negative modulator pictures in the present study.

In addition, the reaction times to the understanding of the solutions were not influenced by the memory of modulator pictures; the positive modulator pictures led to faster reaction times to report understanding the solutions, but the negative modulator pictures increased the response times, regardless of whether participants remembered or forgot those modulator pictures in a subsequent memory test. Similarly, in Study 2, we found increased activity in the angular gyrus and MPFC following positive images, irrespective of whether those images were remembered or forgotten in the subsequent memory test. These results again suggest that the effects of emotional pictures on understanding of insight solutions do not depend on the mechanisms underlying the effects of emotional pictures on memory (which is modulated by arousal). However, given the relatively small number of trials after we categorized remembered versus forgotten items for each valence category, further research should employ more trials to address the relationships between the effects of emotional pictures on memory and those on processing solutions to insight problems.

\section{Questions for future studies}

Several other questions also remain for further work. A first question concerns mood induction procedures. In the present study, we manipulated participants' moods by showing IAPS pictures (Lang et al., 1997) and did not employ other affect induction procedures. This could raise the concern that the effects we observed might be due to confounding factors with this specific affect induction procedure, rather than to participants' mood states. However, the results of the present study are consistent with those of many behavioral studies that have demonstrated facilitative effects of positive moods on insight task performance with different affect induction procedures (e.g., watching positive films, giving small gifts, listening to positive music, and generating positive thoughts; Isen et al., 1987; Rowe et al., 2007). In past studies, researchers have also reported that negative moods impair cognitive flexibility, employing different affect induction procedures (e.g., baseline negative mood states; situational stressors; Alexander et al., 2007; Beversdorf et al., 1999; Subramaniam et al., 2009). In addition, in Study 2, we found that the brief viewing of positive images induced greater activity in MPFC, whose activity is known to be enhanced by sustained positive mood states during insight tasks (Subramaniam et al., 2009). Given these converging results, it seems plausible that the effects of emotional images revealed in the present study are attributable to emotional states instead of other confounding factors. However, it is still possible that brief encounters with other types of emotional stimuli (e.g., sounds, words) might have different influences on cognitive flexibility. Further studies with different emotional modulators are needed to address this question.

Second, in the present study, we employed only one type of insight problems (i.e., riddles). In addition, our participants were given the solutions to riddles, and did not have to actually solve problems. These procedures helped us to reduce the durations for each trial and to increase the number of trials, allowing for manipulating emotional states as a within-participant factor. However, the present procedure is not a common way used in past studies to examine the effects of mood on insight tasks or cognitive flexibility. In addition, riddles used in the present study might be easier than insight problems used in other studies (e.g., Duncker's candle task). Thus, there is a possibility that brief presentation of positive stimuli would have different impacts on insight task performance if tasks are more difficult or if people have to solve those problems by themselves. Furthermore, we measured how long it took participants to report having understood the riddle solutions and did not obtain any objective measures of understanding of the solutions. Given that the results of Study 2 revealed relationships between this reaction time measure and activities in the angular gyrus, which has been implicated in processing of insight tasks (Aziz-Zadeh et al., 2009; Bechtereva et al., 2004; Fink et al., 2009; Jung et al., 2010; Luo et al., 2004), it seems plausible that our reaction time measure reflects cognitive processing involved in understanding of insight solutions. However, there may be other factors that influence reaction times, such as motivations to please the experimenters or simple errors in buttonpress. Further studies should employ different types of insight problems and obtain more detailed measures to elucidate whether and how emotional states affect performance on insight problems.

\section{Conclusions}

In summary, in the present study, we demonstrated that the brief presentation of positive and negative images has different effects on understanding of the solutions to problems requiring insight. Whereas participants were slower to report that they understood the solutions following negative images, they showed faster reaction 
times to report having understood the solutions following positive images. These results suggest that cognitive flexibility is modulated not only by sustained mood states, as shown in previous studies (Isen \& Daubman, 1984; Isen et al., 1987, 1985; Rowe et al., 2007; Subramaniam et al., 2009), but also by simply viewing emotional stimuli for short time periods. In addition, we found that the brief viewing of positive pictures increased activity in the angular gyrus and MPFC during processing of solutions, and that the greater activity in the angular gyrus is associated with faster reaction times to report understanding the solutions. Given that similar brain regions have been implicated in the effects of sustained mood on insight problem solving (Subramaniam et al., 2009), these results suggest that brief viewing of emotional images influences performance on subsequent tasks requiring cognitive flexibility by similar mechanisms to long-lasting emotional states.

Acknowledgement The authors thank Mara Mather, Matthew Sutherland, and Borah Lee for their comments on an earlier version of this manuscript, and Saea Ida for her help in data collection. Part of this study was supported by the NPO Neurocreative Laboratory, a Grant-inAid for Scientific Research (20240026), and a Grant-in-Aid for JSPS Fellow (19-8978).

\section{References}

Alexander, J. K., Hillier, A., Smith, R. M., Tivarus, M. E., \& Beversdorf, D. Q. (2007). Beta-adrenergic modulation of cognitive flexibility during stress. Journal of Cognitive Neuroscience, 19, 468-478. doi:10.1162/jocn.2007.19.3.468

Anderson, A. K. (2005). Affective influences on the attentional dynamics supporting awareness. Journal of Experimental Psychology. General, 134, 258-281. doi:10.1037/00963445.134.2.258

Anderson, A. K., Christoff, K., Stappen, I., Panitz, D., Ghahremani, D. G., Glover, G., et al. (2003). Dissociated neural representations of intensity and valence in human olfaction. Nature Neuroscience, 6, 196-202. doi:10.1038/nn1001

Aquino, J. M., \& Arnell, K. M. (2007). Attention and the processing of emotional words: Dissociating effects of arousal. Psychonomic Bulletin \& Review, 14, 430-435. doi:10.3758/BF03194084

Arnell, K. M., Killman, K. V., \& Fijavz, D. (2007). Blinded by emotion: Target misses follow attention capture by arousing distractors in RSVP. Emotion, 7, 465-477. doi:10.1037/15283542.7.3.465

Aziz-Zadeh, L., Kaplan, J. T., \& Iacoboni, M. (2009). "Aha!": The neural correlates of verbal insight solutions. Human Brain Mapping, 30, 908-916. doi:10.1002/hbm.20554

Baas, M., De Dreu, C. K. W., \& Nijstad, B. A. (2008). A metaanalysis of 25 years of mood-creativity research: Hedonic tone, activation, or regulatory focus? Psychological Bulletin, 134, 779806. doi:10.1037/a0012815

Baumann, N., \& Kuhl, J. (2005). Positive affect and flexibility: Overcoming the precedence of global over local processing of visual information. Motivation and Emotion, 29, 123-134. doi:10.1007/s11031-005-7957-1
Bechtereva, N. P., Korotkov, A. D., Pakhomov, S. V., Roudas, M. S., Starchenko, M. G., \& Medvedev, S. V. (2004). Pet study of brain maintenance of verbal creative activity. International Journal of Psychophysiology, 53, 11-20. doi:10.1016/j.ijpsy cho.2004.01.001

Beversdorf, D. Q., Hughes, J. D., Steinberg, B. A., Lewis, L. D., \& Heilman, K. M. (1999). Noradrenergic modulation of cognitive flexibility in problem solving. Neuroreport, 10, 2763-2767.

Bolte, A., Goschke, T., \& Kuhl, J. (2003). Emotion and intuition: Effects of positive and negative mood on implicit judgments of semantic coherence. Psychological Science, 14, 416-421. doi:10.1111/1467-9280.01456

Botvinick, M. M., Cohen, J. D., \& Carter, C. S. (2004). Conflict monitoring and anterior cingulate cortex: An update. Trends in Cognitive Sciences, 8, 539-546. doi:10.1016/j.tics.2004.10.003

Bowden, E. M., Jung-Beeman, M., Fleck, J., \& Kounios, J. (2005). New approaches to demystifying insight. Trends in Cognitive Sciences, 9, 322-328. doi:10.1016/j.tics.2005.05.012

Brett, M., Anton, J. L., Valabregue, R., \& Poline, J. B. (2002, June). Region of interest analysis using an spm toolbox. Paper presented at the 8th International Conference on Functional Mapping of the Human Brain, Sendai, Japan

Cabeza, R., Ciaramelli, E., Olson, I. R., \& Moscovitch, M. (2008). The parietal cortex and episodic memory: An attentional account. Nature Reviews Neuroscience, 9, 613-625. doi:10.1038/nrn2459

Calvo, M. G., \& Castillo, M. D. (2005). Foveal vs. parafoveal attention-grabbing power of threat-related information. Experimental Psychology, 52, 150-162. doi:10.1027/16183169.52.2.150

Canli, T., Zhao, Z., Brewer, J., Gabrieri, J. D. E., \& Cahill, L. (2000). Event-related activity in the human amygdala associates with later memory for individual emotional experience. Journal of Neuroscience, 20, RC99.

Chambers, C. D., Payne, J. M., Stokes, M. G., \& Mattingley, J. B. (2004). Fast and slow parietal pathways mediate spatial attention. Nature Neuroscience, 7, 217-218. doi:10.1038/nn1203

Corbetta, M., Kincade, J. M., \& Shulman, G. L. (2002). Neural systems for visual orienting and their relationships to spatial working memory. Journal of Cognitive Neuroscience, 14, 508523. doi:10.1162/089892902317362029

Davis, M. A. (2009). Understanding the relationship between mood and creativity: A meta-analysis. Organizational Behavior and Human Decision Processes, 108, 25-38. doi:10.1016/j.obhdp.2008.04.001

Dietrich, A., \& Kanso, R. (2010). A review of EEG, ERP, and neuroimaging studies of creativity and insight. Psychological Bulletin, 136, 822. doi:10.1037/a0019749

Dolcos, F., \& Denkova, E. (2008). Neural correlates of encoding emotional memories: A review of functional neuroimaging evidence. Cell Science Reviews, 5, 78-122.

Dolcos, F., Kragel, P., Wang, L., \& McCarthy, G. (2006). Role of the inferior frontal cortex in coping with distracting emotions. Neuroreport, 17, 1591-1594. doi:10.1097/01.wnr.0000236860.24081.be

Dolcos, F., LaBar, K. S., \& Cabeza, R. (2004). Interaction between the amygdala and the medial temporal lobe memory system predicts better memory for emotional events. Neuron, 42, 855-863. doi:10.1016/S0896-6273(04)00289-2

Dolcos, F., \& McCarthy, G. (2006). Brain systems mediating cognitive interference by emotional distraction. Journal of Neuroscience, 15, 2072-2079. doi:10.1523/JNEUROSCI.5042-05.2006

Dreisbach, G. (2006). How positive affect modulates cognitive control: The costs and benefits of reduced maintenance capability. Brain and Cognition, 60, 11-19. doi:10.1016/j.bandc.2005.08.003

Dreisbach, G., \& Goschke, T. (2004). How positive affect modulates cognitive control: Reduced perseveration at the cost of increased distractibility. Journal of Experimental Psychology. Learning, 
Memory, and Cognition, 30, 343-353. doi:10.1037/02787393.30.2.343

Fenske, M. J., \& Eastwood, J. D. (2003). Modulation of focused attention by faces expressing emotion: Evidence from flanker tasks. Emotion, 3, 327-343. doi:10.1037/1528-3542.3.4.327

Fink, A., Grabner, R. H., Benedek, M., Reishofer, G., Hauswirth, V., Fally, M., et al. (2009). The creative brain: Investigation of brain activity during creative problem solving by means of EEG and fMRI. Human Brain Mapping, 30, 734-748.

Fredrickson, B. L. (2004). The broaden-and-build theory of positive emotions. Philosophical Transactions of the Royal Society B: Biological Sciences, 359, 1367-1377. doi:10.1098/ rstb.2004.1512

Fredrickson, B. L., \& Branigan, C. (2005). Positive emotions broaden the scope of attention and thought-action repertoires. Cognition and Emotion, 19, 313-332. doi:10.1080/02699930441000238

Gasper, K., \& Clore, G. L. (2002). Attending to the big picture: Mood and global versus local processing of visual information. Psychological Science, 13, 34-40. doi:10.1111/1467-9280.00406

Hamann, S., Ely, T. D., Grafton, S. T., \& Kilts, C. D. (1999). Amygdala activity related to enhanced memory for pleasant and aversive stimuli. Nature Neuroscience, 2, 289-293. doi:10.1038/ 6404

Ihssen, N., Heim, S., \& Keil, A. (2007). The costs of emotional attention: Affective processing inhibits subsequent lexicosemantic analysis. Journal of Cognitive Neuroscience, 19, 1932-1979. doi:10.1162/jocn.2007.19.12.1932

Ihssen, N., \& Keil, A. (2009). The costs and benefits of processing emotional stimuli during rapid serial visual presentation. Cognition and Emotion, 23, 296-326. doi:10.1080/ 02699930801987504

Indovina, I., \& Macaluso, E. (2007). Dissociation of stimulus relevance and saliency factors during shifts of visuospatial attention. Cerebral Cortex, 17, 1701-1711. doi:10.1093/cercor/ bh1081

Isen, A., \& Daubman, K. A. (1984). The influence of affect on categorization. Journal of Personality and Social Psychology, 47, 1206-1217. doi:10.1037/0022-3514.47.6.1206

Isen, A., Daubman, K. A., \& Nowicki, G. P. (1987). Positive affect facilitates creative problem solving. Journal of Personality and Social Psychology, 52, 1122-1131. doi:10.1037/00223514.52.6.1122

Isen, A., Johnson, M. M. S., Mertz, E., \& Robinson, G. F. (1985). The influence of positive affect on the unusualness of word associations. Journal of Personality and Social Psychology, 48, 1413-1426. doi:10.1037/0022-3514.48.6.1413

Jung-Beeman, M., Bowden, E. M., Haberman, J., Frymiare, J. L., Arambel-Liu, S., Greenblatt, R., et al. (2004). Neural activity when people solve verbal problems with insight. PLoS Biology, 2, E97. doi:10.1371/journal.pbio.0020097

Jung, R. E., Segall, J. M., Jeremy Bockholt, H., Flores, R. A., Smith, S. M., Chavez, R. S., et al. (2010). Neuroanatomy of creativity. Human Brain Mapping, 31, 398-409. doi:10.1016/j.neuropsy chologia.2005.02.013

Kensinger, E. A., \& Corkin, S. (2003). Effect of negative emotional content on working memory and long-term memory. Emotion, 3, 378-393. doi:10.1037/1528-3542.3.4.378

Kensinger, E. A., \& Corkin, S. (2004). Two routs to emotional memory: Distinct neural processes for valence and arousal. Proceedings of the National Academy of Science, 101, 3310 3315. doi:10.1073/pnas.0306408101

Kunosis, J., Frymiare, J. L., Bowden, E. M., Fleck, J., Subramaniam, K., Parrish, T. B., et al. (2006). The prepared mind: Neural activity prior to problem presentation predicts solution by sudden insight. Psychological Science, 17, 882-890.
Lancaster, J. L., Tordesillas-Gutierrez, D., Martinez, M., Salinas, F., Evans, A., Zilles, K., et al. (2007). Bias between MNI and talairach coordinates analyzed using the icbn-152 brain template. Human Brain Mapping, 28, 1194-1205. doi:10.1002/hbm.20345

Lancaster, J. L., Woldorff, M. G., Parsons, L. M., Liotti, M., Freitas, C. S., Rainey, L., et al. (2000). Automated talairach atlas labels for functional brain mapping. Human Brain Mapping, 10, 120-131. doi:10.1002/1097-0193(200007)10:3<120::AID-HBM30>3.0. $\mathrm{CO} ; 2-8$

Lang, P. J., Bradley, M. M., \& Cuthbert, B. N. (1997). International affective picture system (IAPS): Technical manual and affective ratings. NIMH center for the study of emotion and attention

Luo, J., \& Knoblich, G. (2007). Studying insight problem solving with neuroscientific methods. Methods, 42, 77-86. doi:10.1016/j. ymeth.2006.12.005

Luo, J., \& Niki, K. (2003). Function of hippocampus in "insight" of problem solving. Hippocampus, 13, 316-323. doi:10.1002/ hipo.10069

Luo, J., Niki, K., \& Knoblich, G. (2006). Perceptual contributions to problem solving: Chunk decomposition of chinese characters. Brain Research Bulletin, 70, 430-443. doi:10.1016/j.brainres bull.2006.07.005

Luo, J., Niki, K., \& Phillips, S. (2004). Neural correlates of the 'aha! Reaction'. Neuroreport, 15, 2013-2017.

MacLeod, C. M., \& MacDonald, P. A. (2000). Interdimensional interference in the stroop effect: Uncovering the cognitive and neural anatomy of attention. Trends in Cognitive Sciences, 4, 383-391. doi:10.1016/S1364-6613(00)01530-8

Mai, X. Q., Luo, J., Wu, J. H., \& Luo, Y. J. (2004). “Aha!” effects in a guessing riddle task: An event-related potential study. Human Brain Mapping, 22, 261-270. doi:10.1002/hbm.20030

Mather, M., Mitchell, K. J., Raye, C. L., Novak, D. L., Greene, E. J., \& Johnson, M. K. (2006). Emotional arousal can impair feature binding in working memory. Journal of Cognitive Neuroscience, 18, 614-625. doi:10.1162/jocn.2006.18.4.614

Mather, M., \& Sutherland, M. (2009). Disentangling the effects of arousal and valence on memory for intrinsic details. Emotion Review, 1, 118-119. doi:10.1177/1754073908100435

McKenna, F. P., \& Sharma, D. (1995). Intrusive cognitions: An investigation of the emotional stroop task. Journal of Experimental Psychology. Learning, Memory, and Cognition, 21, 15951607. doi:10.1037/0278-7393.21.6.1595

Most, S. B., Chun, M. M., Widders, D. M., \& Zald, D. H. (2005). Attentional rubbernecking: Cognitive control and personality in emotion-induced blindness. Psychonomic Bulletin \& Review, 12, 654-661. doi:10.3758/BF03196754

Nachev, P., Rees, G., Parton, A., Kennard, C., \& Husain, M. (2005). Volition and conflict in human medial frontal cortex. Current Biology, 15, 122-128. doi:10.1016/j.cub.2005.01.006

Qiu, J., Li, H., Luo, Y., Chen, A. T., Zhang, F., Zhang, J., et al. (2006). Brain mechanism of cognitive conflict in a guessing chinese logogriph task. Neuroreport, 17, 679.

Qiu, J., Li, H., Yang, D., Luo, Y., Li, Y., Wu, Z., et al. (2008). The neural basis of insight problem solving: An event-related potential study. Brain and Cognition, 68, 100-106. doi:10.1016/ j.bandc. 2008.03 .004

Raudenbush, S. W., \& Bryk, A. S. (2002). Hierarchical linear models (2nd ed.). Thousand Oaks: Sage.

Ridderinkhof, K. R., Ullsperger, M., Crone, E. A., \& Nieuwenhuis, S. (2004). The role of the medial frontal cortex in cognitive control. Science, 306, 443-447. doi:10.1126/science.1100301

Rowe, G., Hirsh, J. B., \& Anderson, A. K. (2007). Positive affect increases the breadth of attentional selection. Proceedings of National Academy of Sciences, 104, 383-388. doi:10.1073/ pnas.0605198104 
Rudrauf, D., Lachaux, J.-P., Damasio, A. R., Baillet, S., Hugueville, L., Martinerie, J., et al. (2009). Enter feelings: Somatosensory responses following early stages of visual induction of emotion. International Journal of Psychophysiology, 72, 13-23. doi:10.1016/j.ijpsycho.2008.03.015

Schimmack, U. (2005). Attentional interference effects of emotional pictures: Threat, negativity, or arousal? Emotion, 5, 55-66. doi:10.1037/1528-3542.5.1.55

Schmitz, T. W., De Rosa, E., \& Anderson, A. K. (2009). Opposing influences of affective state valence on visual cortical encoding. Journal of Neuroscience, 29, 7199-7207. doi:10.1523/jneuro sci.5387-08.2009

Shibata, T., Tanikawa, S., \& Yagawa, S. (1984). World encyclopedia of riddles. Tokyo: Taishu-kan.
Subramaniam, K., Kounios, J., Parrish, T. B., \& Jung-Beeman, M. (2009). A brain mechanism for facilitation of insight by positive affect. Journal of Cognitive Neuroscience, 21, 415-432. doi:10.1162/jocn.2009.21057

Vosburg, S. K. (1998). The effects of positive and negative mood on divergent-thinking performance. Creativity Research Journal, 11, 165-172. doi:10.1207/s15326934crj1102 6

Weisberg, R. W. (1996). Prolegomena to theories of insight in problem solving: A taxonomy of problems. In R. J. Sternberg \& J. E. Davidson (Eds.), The nature of insight (pp. 157-196). Cambridge: MIT Press.

Worsley, K. J. (2001). Statistical analysis of activation images. In J. Jezzard, P. M. Matthews, \& S. M. Smith (Eds.), Functional MRI: An introduction to methods (pp. 251-270). New York: Oxford University Press. 